\title{
Insights into the Complex Prebreakdown Actuation of Silicone Elastomers and its
} Influence on Breakdown Behavior

\author{
Vaicekauskiate, Justina; Yu, Liyun; Skov, Anne Ladegaard
}

\section{Published in: \\ ACS Omega}

Link to article, DOI:

10.1021/acsomega.0c00785

Publication date:

2020

Document Version

Publisher's PDF, also known as Version of record

Link back to DTU Orbit

Citation (APA):

Vaicekauskiate, J., Yu, L., \& Skov, A. L. (2020). Insights into the Complex Prebreakdown Actuation of Silicone Elastomers and its Influence on Breakdown Behavior. ACS Omega, 5(30), 18584-18593.

https://doi.org/10.1021/acsomega.0c00785

\section{General rights}

Copyright and moral rights for the publications made accessible in the public portal are retained by the authors and/or other copyright owners and it is a condition of accessing publications that users recognise and abide by the legal requirements associated with these rights.

- Users may download and print one copy of any publication from the public portal for the purpose of private study or research.

- You may not further distribute the material or use it for any profit-making activity or commercial gain

- You may freely distribute the URL identifying the publication in the public portal 


\title{
Insights into the Complex Prebreakdown Actuation of Silicone Elastomers and its Influence on Breakdown Behavior
}

\author{
Justina Vaicekauskiate, Liyun Yu, and Anne Ladegaard Skov*
}

Cite This: ACS Omega 2020, 5, 18584-18593

Read Online

ABSTRACT: Dielectric elastomer transducers can be applied in many different applications, but their current use is limited by either their electrical breakdown strength or by electromechanical instabilities in the case of soft elastomers. The breakdown process is never a single, simple process but rather-most likely-an ensemble of thermoelectric processes taking place in
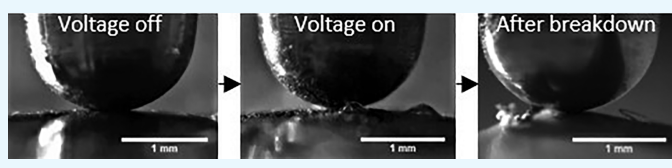
both elastomer and electrode materials, coupled with mechanical and potentially also chemical degradation. In this work, by using a high-speed camera, we follow silicone-based dielectric elastomers undergoing a ramp-up in voltage close to electrical breakdown strength, with differently constructed elastomers and electrodes. As such, we present experimental insights into the electromechanical processes immediately before the dielectric breakdown of elastomers and identify three different actuation mechanisms taking place prior to electrical breakdown, denoted prebreakdown actuation in the following. The prebreakdown actuation mechanisms observed herein include film thinning and stretching, as well as the formation of bubble- and ring-shaped structures from the elastomer surface, respectively. We furthermore present a theoretical explanation for the observed actuation mechanisms.

\section{INTRODUCTION}

Dielectric elastomer transducers (DETs) are prepared by placing an electrically deformable elastomer between two compliant electrodes. When a DET converts electrical energy into mechanical energy, it is called a dielectric elastomer actuator (DEA). In actuation mode, a voltage is applied over the electrodes, and the dielectric elastomer actuator expands as a result of the electroattraction between the two opposing electrodes and the electrorepulsion of similar charges on the individual respective electrodes. ${ }^{1-4}$ This electric deformation has been known since the 18th century, but dielectric elastomers have been studied to any great extent for only the past 30 years, due to the breakthrough in compliant electrodes that enabled the technology. ${ }^{5}$ In the late 19th century, Röntgen performed simple experiments by charging the surfaces of elastomers, thereby proving actuation. 6,7

DEAs can be used for soft robotics, ${ }^{8,9}$ tactile and haptic devices, ${ }^{10,11}$ and optical systems, ${ }^{12}$ among many others. ${ }^{13}$ However, their use is usually limited by their inherent electrical breakdown strength or electromechanical instability, causing breakdown. ${ }^{14,15}$ The fundamentals behind the electrical breakdown of these thin and elastic films are still neither currently fully understood nor elucidated. Static breakdown tests of films between two rigid electrodes have been used extensively over many years to evaluate the stability, for example, of insulating polymers in high electrical fields and are still gaining importance, due to an increasing demand for novel polymeric materials applied in high-voltage applications. ${ }^{16}$ However, the recommended testing for electrical breakdown strength is static, ${ }^{17,18}$ while for inherently soft dielectric elastomers, electrical breakdown is far from static. Therefore, coupling between static and dynamic measurements is needed, to ensure a more reliable determination of the breakdown strength of the dielectric elastomer actuator.

There exist several theoretical models assessing the underlying physical or chemical phenomena occurring during the dielectric elastomer breakdown process, and the most discussed and best understood phenomenon is pull-in instability, ${ }^{19-28}$ which depends on elastomer properties (mechanical and electrical) along with the type of electrode and the experimental setup used for actuation. When a voltage is applied to an unconstrained sample, the elastomer undergoes a local thinning process, following which the applied voltage will cause a locally higher electrical field and thereby amplify this thinning. If the elasticity of the sample cannot be balanced by inherent strain-hardening of the elastomer, thinning continues and leads to pull-in instability. In contrast, when the same sample is constrained, for example, by a rigid frame, there will be no drastic thinning of the sample; instead, out-ofplane deformations may take place such that the entire surface of the elastomer wrinkles. This wrinkling process usually leads to pull-in instability, due to the inhomogeneous fields created and the subsequent breakdown. ${ }^{20,24,29-31}$

Received: February 22, 2020

Accepted: July 7, 2020

Published: July 20, 2020 
Table 1. Breakdown Strengths of the Different Samples for the Different Experimental Setups $(\mathrm{V} / \mu \mathrm{m})^{a}$

\begin{tabular}{|c|c|c|c|c|c|c|c|c|c|c|}
\hline \multirow[b]{3}{*}{ sample number } & \multicolumn{4}{|c|}{ no prestretch } & \multicolumn{3}{|c|}{ uniaxial prestretch } & \multicolumn{3}{|c|}{ biaxial prestretch } \\
\hline & \multirow[b]{2}{*}{ electrode A } & \multirow[b]{2}{*}{ electrode B } & \multirow[b]{2}{*}{ electrode C } & \multirow[b]{2}{*}{ electrode D } & \multicolumn{3}{|c|}{ electrode $\mathrm{C}$} & \multicolumn{3}{|c|}{ electrode C } \\
\hline & & & & & $10 \%$ & $20 \%$ & $100 \%$ & $10 \%$ & $20 \%$ & $100 \%$ \\
\hline 1 & $145 \pm 33$ & $181 \pm 2$ & $181 \pm 17$ & $136 \pm 9$ & $191 \pm 14$ & $179 \pm 5$ & $296^{*}$ & $220 \pm 8$ & $272 \pm 43$ & $416^{*}$ \\
\hline 2 & $116 \pm 26$ & $185 \pm 33$ & $159 \pm 7$ & $158 \pm 12$ & $142 \pm 32$ & $148 \pm 30$ & $233 *$ & $140 \pm 6$ & $209 \pm 22$ & $422 *$ \\
\hline 3 & $120 \pm 5$ & $123 \pm 17$ & $129 \pm 5$ & $129 \pm 12$ & $130 \pm 3$ & $124 \pm 8$ & $194 \pm 4$ & $139 \pm 3$ & $159 \pm 13$ & $447 \pm 54$ \\
\hline
\end{tabular}

$a_{*}$ Prestretched sample is too brittle; measurement carried out successfully for one sample only.

Also, more recently, studies investigating a possible thermal runaway leading to electrical breakdown have been conducted. However, neither thermal runaway nor pull-in can explain why dielectric elastomers not always break down in the area where the electrical field is the largest. ${ }^{32,33}$ Herein, we present some of the mechanical actuation processes occurring for thin dielectric elastomer films when a voltage is applied in experimental setups with fewer and more restricted configurations to investigate what leads to breakdowns outside the highest electrical field. In addition, the underlying theory behind these methods is discussed. To evaluate the actuation processes, a high-speed camera is coupled with the electrical breakdown measurement, to capture the macroscopic activities taking place during dielectric breakdown. We use electrodes with different aspect ratios and dimensions to investigate their effect on the breakdown mechanisms. Recorded videos are analyzed, and then three main types of structures are identified as they appear. The shape, size, and location of the structures are investigated, and breakdown location is also reported, since it does not necessarily occur in either the area where actuation takes place or where the field is the largest.

\section{RESULTS AND DISCUSSION}

2.1. Breakdown Strength. The electrical breakdown strengths resulting from the different experimental setups are presented in Table 1, from which it is evident that breakdown strength varies significantly depending on electrode type (Table 4), albeit there are no obvious trends. Nevertheless, sample quality depends on microvoids, deviations in film thickness, surface roughness, dust particles, and scratches that may be found in a sample, all of which can cause irregular sample performance when the voltage is applied and large deviations in breakdown strength. ${ }^{25,34,35}$ A general trend, however, is observed when it comes to improving breakdown strength, following on from prestretching. This is a well-known and common effect, but the exact origin remains vague and may vary from elastomer to elastomer; ${ }^{36}$ nonetheless, possible reasons are the orientation of polymer chains, ${ }^{2,37}$ stretch-out of film defects, ${ }^{38}$ and strain-hardening effects. ${ }^{36}$

2.2. Film Sample Actuation before Breakdown. It is generally known that when a voltage is applied to a DEA, the elastomer starts to actuate, ${ }^{1-4}$ to balance the effect of electrostatic attraction with the compressive forces of the elastomer. The charging and decharging of electrodes are usually very fast such that actuation appears instantaneously, and when the voltage reaches a critical value that can no longer be balanced by elasticity, the elastomer breaks down. $7,23,39$ Here, we show that more complex actuation mechanisms take place and are not limited to sample thinning. The actuation mechanisms presented herein are combinations of film stretching and the formation of bubbles or ring-shaped structures, and they are now discussed in further detail. The distance from the electrode and the size of bubble structures are also investigated. Results are, however, inconclusive due to the difficulty in determining the characteristic of this very dynamic process. Data are given in Supporting Information Table S2.

Film stretching in the strongest electrical field in between the electrodes is observed in most experiments. The initial actuation mechanism occurring when a voltage is applied involves film thinning, before the film starts to stretch due to the incompressibility of the elastomer, and finally, the film surrounds the lower electrode. This behavior can be seen in Supporting Information Video 1 and in Figure 1, where the

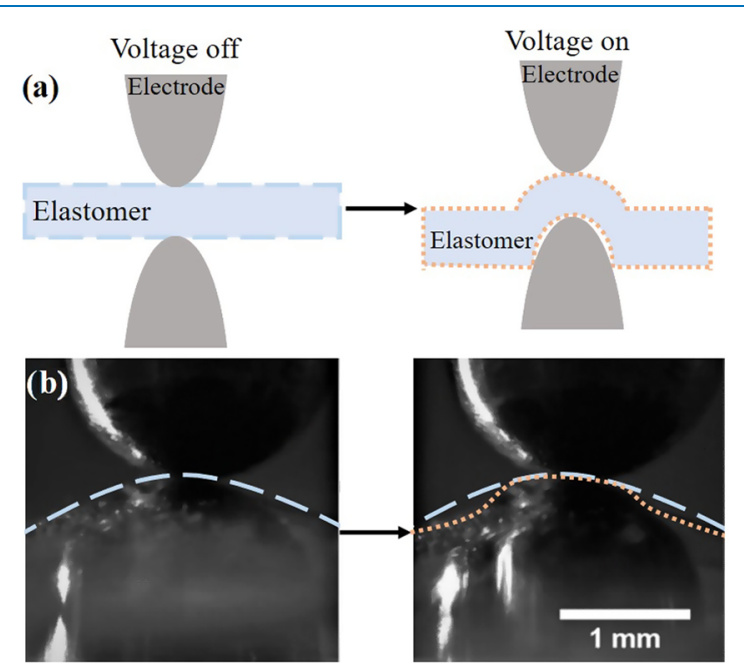

Figure 1. Sample thinning and stretching actuation mechanism occurring in the beginning of the voltage ramp-up. The blue dashed line represents the contour of the film sample before actuation. The orange dotted line represents the contour of an actuated sample. (a) Sample stretching scheme. Initially, when the voltage is applied, the film stretches and surrounds the lower electrode. (b) Digital photo of sample 1 actuating on the small $(2 \mathrm{~mm})$ electrode.

sample starts to thin under the influence of gravity and ends up resting on the lower electrode. This type of actuation (thinning and stretching) is negligible and notable only in fast-forwarded video recording.

When the voltage is increased further, the film starts to move upward, following which it bends and then finally forms bubbles. This actuation mechanism is referred to herein as the "bubble structure" and is presented in Figure 2 and Supporting Information Video 2. Bubble structure diameters vary in size, depending on which type of electrode is used, but usually, it is between 0.7 and $3 \mathrm{~mm}$. Bubble structures are observed just before the breakdown, when small, small + large, large, or flat electrodes are used with samples 1 and 2. This actuation is not observed with wire or sputter-coated electrodes, or in sample 3, 


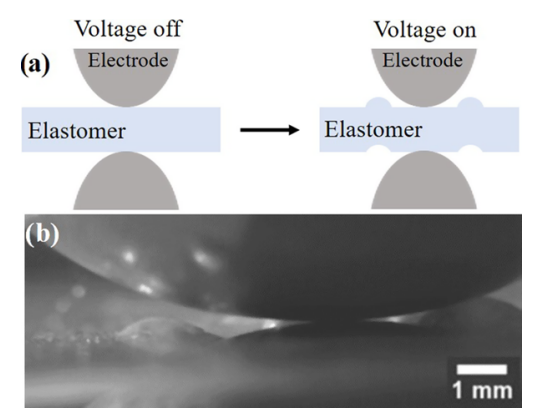

Figure 2. Bubble structure actuation mechanism. (a) Schematics of a sample forming bubbles. (b) Digital photo of bubble structures resulting from the large $(2 \mathrm{~cm})$ electrode in sample 2 .

which mainly undergoes film stretching, as discussed before, and does not perform any other actuation processes. We hypothesize that small variations in the sample quality also plays an important role in the prebreakdown actuation process, as also pointed out by Silau et al. from another experimental study. ${ }^{36}$ When wire electrodes are used, all samples are sputtercoated with gold. The actuation of sputter-coated samples is more pronounced, since a much larger area is affected by the electrical field, and bending of the very flexible elastomers does not require much energy. For that reason, samples tested with wire electrodes have greater actuation, which is explained further in the following section.

An alternative pattern is observed when the large electrode is used or samples are coated with gold. This pattern is observed when a larger sample area is exposed to the high electrical field (because of the larger semispherical-shaped electrode or the gold layer). A ring-shaped structure is formed as a result of a few smaller bubbles interconnecting and encircling the upper electrode, an example of which is presented in Figure 3 and Supporting Information Video 3. The transition from bubbles to ring shape is very fast.

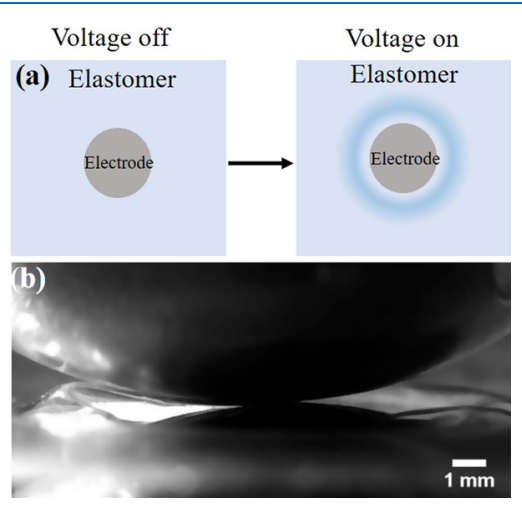

Figure 3. Ring-shaped actuation mechanism. (a) Schematic top view of a sample forming a ring structure around the electrode. (b) Digital photo of a ring structure resulting from a $2 \mathrm{~cm}$ electrode, using sample 1.

To make sure that the resulting structures are not caused by solvent or impurity evaporation within the film (such as absorbed water by the silica particles and/or residual monomer and solvent), leading to cavities just below the elastomer surface, the samples are additionally postcured at $80{ }^{\circ} \mathrm{C}$ for 70 $\mathrm{h}$ and then retested. This postcuring procedure has been proven sufficient for ensuring the full removal of excess solvent and other residuals for thin silicone elastomer films. ${ }^{34}$ After postcuring, the same breakdown experiments are carried out and no change in the bubble and ring structures is observed. As a result, we can conclude that the bubbles are not the result of solvent evaporation, and film actuation is caused solely by electromechanical and electrostatic forces. Furthermore, by placing double-sided adhesive tape on the lower flat electrode, in this way constraining the film sample in the plane perpendicular to the electrical field, it is determined that actuation during the breakdown tests results from sample bending and moving closer to the electrode, but not in the presence of overall sample expansion during actuation since there is no difference in behaviors from fully fixed and regularly placed samples.

Some common observations are discussed in the following. Naturally, all types of structures disappear as soon as breakdown occurs, and bubble- or ring-shaped structures never directly touch the electrode. Counterintuitively, breakdown for non-prestretched samples never takes place under the electrode, where the field concentration is the highest (Figure $9 \mathrm{~b})$, or in the bubble or ring structure, where film instability is the highest. On the other hand, breakdowns are not encountered around the edges of geometries either, so we can also eliminate the effect of high fields around the edges of the applied electrodes, etc. The resulting breakdown pinhole is observed to be located randomly across the testing area, which we ascribe to local film imperfections. When samples are prestretched, breakdown always occurs under the electrode where the field is the largest. This observation further strengthens our hypothesis that the breakdown takes place around any present imperfection despite the imperfection being placed away from the strongest field. Breakdown within or around imperfections can be explained by the initial theory posited by Blok and LeGrand, ${ }^{14}$ later refined by Bigué et al., ${ }^{35}$ Zhao and Wang, ${ }^{25}$ and Zakaria et al., ${ }^{34}$ to better describe dielectric elastomers. They considered that electrical fields in dielectric elastomers are not always homogeneous. Inside a dielectric elastomer, there are always some irregular regions, for example, voids or gas bubbles, deviations in a film's thickness or surface roughness from uneven curing or the evaporation of residual byproducts from the polymerization process, or even dust particles as a result of elastomer composition or from processing. When the voltage is applied, these regions are subjected to a higher-than-average electrical field, and a so-called "field enhancement" is observed. In the film's weak spots, the electrical field is even higher and causes a slight deformation of the film; alternatively, in the case of air voids, the air starts breaking down, which leads to sparks. Electrical field enhancement leads to the formation of indentations, and it becomes even more inhomogeneous at the deformation site, meaning that breakdown occurs when breakdown strength is reached at the thinnest spot of the film.

2.3. Film Samples Coated with Gold. The film sample's surface is coated with gold to investigate if actuation is influenced by the active surface area (Table $4 \mathrm{~F}$ ). When a voltage is applied to samples with a gold layer, all of them exhibit greater actuation compared to the uncoated samples. When breakdown occurs, it lasts much longer than for uncoated samples, for which breakdown is instantaneous. The gold electrode samples appear to burn (Supporting Information Video 4). Furthermore, the resulting pinholes from the breakdown are larger, which agrees well with the much higher number of charges going through the pinhole after short-circuiting. 
In addition, when the samples are coated with gold, partial electrical discharges (sparkles) appear more frequently compared to noncoated samples, and after partial discharge occurs, gold-coated samples start to change color. Before the experiment, samples coated with gold have a blue/gray color (Table 4F), but after the partial discharge, some parts of the samples become pink/red in the given region (Figure 4). This

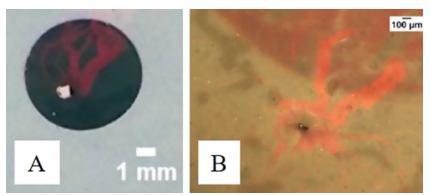

Figure 4. Pink discharge channel marks on the sample. (A) Photo of sample 2 coated with gold and (B) microscope picture of sample 2 coated with gold.

phenomenon can be explained as follows: when the discharge occurs, it creates a channel connecting the electrode with the dielectric elastomer. Electrons from the electrode then search for the easiest way to reach the electrode on the opposite side. The discharge channel causes the temperature and pressure to increase, thus causing gold annealing and a change in color. $^{40,41}$

2.4. Prestretched Samples. As known from previous studies, the prestretching of an incompressible dielectric elastomer decreases its thickness, constrains pull-in instability, and, in this way, increases breakdown strength. ${ }^{3,34,42-45}$ We tested how the actuation mechanism and breakdown phenomena are influenced by prestretching a film sample. Samples are prestretched biaxially and uniaxially, with 10, 20, and $100 \%$ prestretches. It is readily observable that when samples are prestretched, the breakdown pinholes are smaller than their respective pinholes from non-prestretched samples and are always located where the electrical field is the highest (Figure 9b), namely, directly in the middle of the two electrodes. This agrees well with the hypothesis of defects being eliminated by prestretching such that electrical breakdown strength determined as an inherent property of the given elastomer (at the given stretch ratio) rather than-as for the non-prestretched samples-taking place in or nearby a defect. The prestretched samples all tear apart after breakdown (Figure $5 b$ ), but it is observed that prestretching increases sample breakdown strength (Figure 6), as imperfections on a film are stretched out and the sample becomes more homogeneous. We confirm that the introduction of prestretching is a good way to avoid sample actuation mechanisms (stretching and bubble formation or ring-shaped structures)
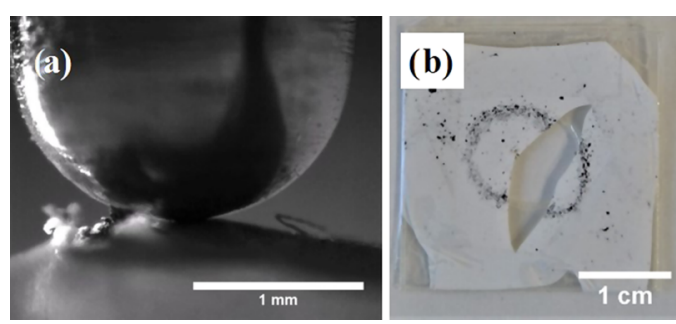

Figure 5. Macroscopically destructed samples 2 after breakdowns. (a) Sample 2 with a pinhole to the left of the electrodes (small electrodes) and (b) $20 \%$ prestretched sample 2 after breakdown, where the breakdown occurred right in the middle of the geometry. and to increase sample breakdown strength. In fact, the improved breakdown strengths may also be a result of the absence of prebreakdown actuation behaviors and not solely on stretching out of imperfections, but data here are not sufficient to conclude this.

When samples are prestretched biaxially by 10 and $20 \%$, film actuation is observed. The films stretch and surround the lower electrode, as shown in Figure 1. However, this actuation is minimal compared to that of the nonstretched samples. With the $100 \%$ biaxial prestretched samples, no actuation is observed, clearly indicating that actuation affects breakdown strength in a negative way despite the breakdown not taking place in the most affected area.

When the samples are prestretched uniaxially, the same actuation as described in Figure 1 is observed, where the sample stretches and surrounds the lower electrode. On rare occasions, just before the breakdown of samples 1 or 2 , bubble formation is observed. We conclude that biaxial prestretching works better on preventing preactuation structure formation.

Experiments with prestretched samples are performed directly after prestretching, $12 \mathrm{~h}$ after prestretching, or 14 days thereafter to see if time and mechanical aging have any influence on the sample actuation mechanism. As discussed earlier, when the elastomer is prestretched, the polymer chains will align in the plane perpendicular to the electrical field. A higher breakdown strength is obtained because of this chain alignment, which prevents charge carrier movements throughout the sample. ${ }^{42}$ If the sample is prestretched and the strain is maintained for a given period of time, the sample starts to undergo mechanical aging and/or a decrease in stress caused by stress relaxation. ${ }^{43}$ This mechanical aging and/or stress relaxation then causes a decrease in breakdown strength. ${ }^{34}$ To test stress relaxation in elastomers, samples are prestretched for different time intervals, prior to the breakdown experiments. One might expect that when the sample is tested after a longer prestretching time, it will have undergone stress relaxation. In that case, the actuation processes, as described earlier, may become more notable for such samples; our results, however, show that there are no differences between samples in terms of different prestretching times, as they all actuated in the same way, i.e., by stretching on the lower electrode.

2.5. Experiment with Wires. To gain a better understanding of how samples actuate when different electrodes are used, gold-coated samples and small wire electrodes are used. When a $5 \mathrm{kV}$ voltage is applied, samples start to actuate and move, curving against the electrical field's direction (Figure 7). The applied voltage is too small to short-circuit the sample, but actuation can still be observed. During actuation, the elastomer sample always curves to the $\mathrm{HV}+$ side (Figure 7), no matter the direction of the gravitational field.

A theoretical explanation of the actuation mechanisms that were described earlier is presented in Figure 8. Electrical field lines are perpendicular to the electrode surface, and due to the latter's shape, the highest electrical field is directly under the electrode (Figure 9b). When the voltage is applied, the sample starts to actuate. The film thins down, then begins to stretch and surrounds the lower electrode. When the voltage is increased, the electrical field surrounding the electrode rises and starts to create a negative corona. Dipoles in the dielectric elastomer between the electrodes are polarized in the direction of the electrical field. However, the negative corona surrounding the electrode causes the dipoles in the elastomer around the electrode to polarize in the opposite direction 

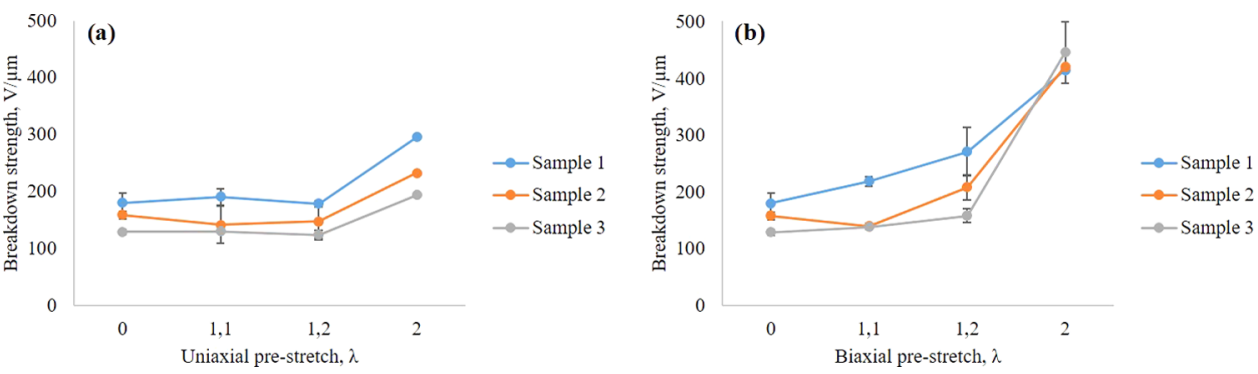

Figure 6. Results of the dielectric breakdown strength of uniaxially (a) and biaxially (b) prestretched samples.

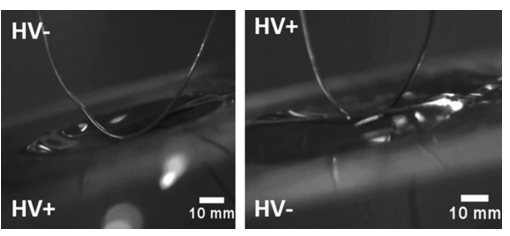

Figure 7. Experiment with wire electrodes. Sample 1, coated with gold. The elastomer sample can be observed curving out in the direction of the $\mathrm{HV}+$ electrode.

(Figure 8, middle). Now the surface of elastomer is positively charged, and thus the elastomer is exposed to electrostatic forces and attracted to the negative corona; subsequently, the film folds (Figure 8, right). Bubble- or ring-shaped structures are observed as a result of this electroactive attraction when dipoles in the elastomer are polarized by a negative corona.

Similar effects were observed on devices with compliant electrodes, and analytical models were presented to describe this phenomenon, denoted electromechanical instability (EMI). ${ }^{4,20,22,30,31,46}$ When the applied voltage is small, the part of the sample covered with electrode expands. When the voltage is increased, wrinkles start to form in some regions due to local defects. When the applied electric field reaches a critical value, the dielectric elastomer undergoes an electromechanical instability and thins down drastically. Thereby, a macroscopically wrinkled pattern on the elastomer surface is formed to release the elastic energy. ${ }^{30,46}$ In our work, due to electrode shape and size, only a small sample area is subjected to the electric field. For that reason, only local wrinkling can be observed, which is expressed as bubble- or ring-shaped structure. However, with the smaller geometry, we can observe local effects and defects.

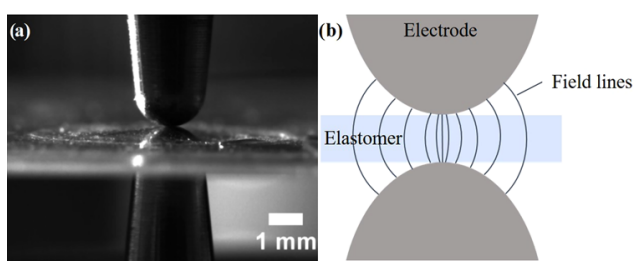

Figure 9. Sample between the electrodes. (a) Sample 3 between two small $(2 \mathrm{~mm})$ electrodes. (b) Scheme for the field lines between two semispherical electrodes. Electrical field concentration is highest in the middle between the electrodes, as indicated by the number of field lines.

\section{CONCLUSIONS}

Breakdown tests have been used for many years to investigate a material's properties when it is exposed to an electrical field; nonetheless, the underlying mechanisms behind this phenomenon are still not fully investigated. Herein, we present insights into the dielectric breakdown of elastomers and what happens prior to this event. We investigated the breakdown strength measurements of silicone elastomers, using three different samples and six different electrode types. The measurements are coupled with video recording with a high-speed camera system. We show that leading up to a breakdown, multiple actuation mechanisms take place when a voltage is applied, the sample is microscopically stretched, and bubble- or ring-shaped structures form. In this work, we show that bubble- or ringshaped structures need to be avoided to have a properly working device. Results of this work confirm that breakdown occurs in the imperfections of a sample. Therefore, for an efficiently working DEA, it is important to synthesize an elastomer of high quality, without any imperfections (such as

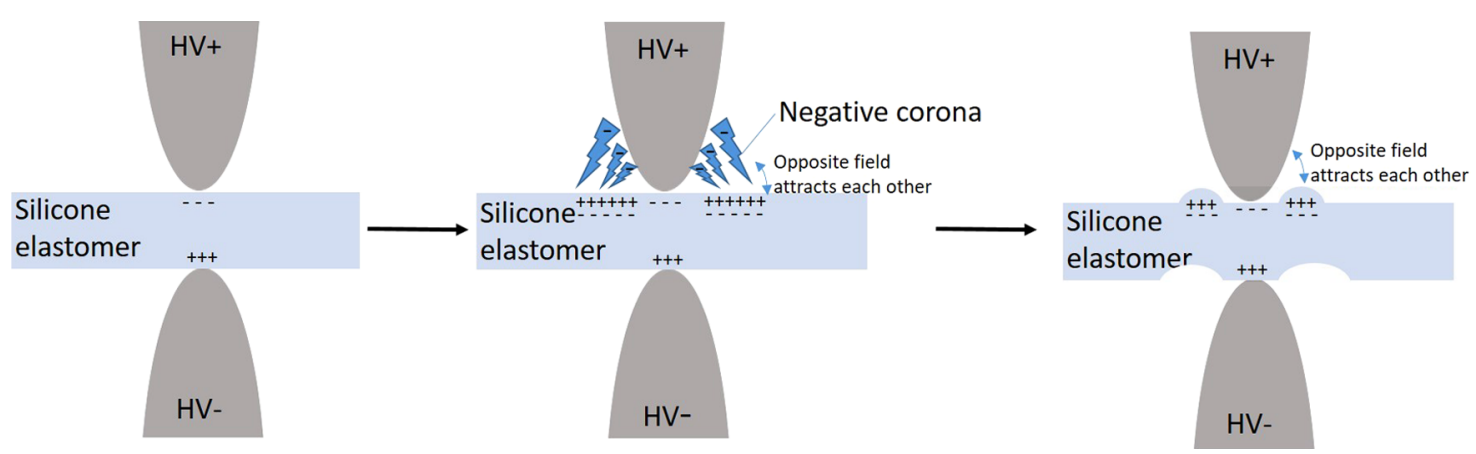

Figure 8. Corona-induced actuation mechanism. The silicone elastomer is placed between two metal electrodes. The field under the electrode polarizes the dipoles in the elastomer in the direction of an electrical field. When the voltage is ramped, the electrical field surrounding the electrode creates a negative corona, which causes dipoles to polarize opposite to the field direction, and the sample surface is charged positively. The elastomer exposed to electrostatic forces folds up to the negative corona, thereby creating bubble- or ring-shaped structures. 
Table 2. Information on Sample Preparation and Resulting Film Thickness

\begin{tabular}{|c|c|c|c|c|c|c|}
\hline \multirow[b]{2}{*}{ sample number } & \multirow[b]{2}{*}{ composition } & \multicolumn{4}{|c|}{ parts } & \multirow[b]{2}{*}{ sample thickness $(\mu \mathrm{m})$} \\
\hline & & LR $3043 \mathrm{~A}$ & LR $3043 \mathrm{~B}$ & $\mathrm{TiO}_{2} / \mathrm{SiO}_{2}$ filler & Belsil solvent & \\
\hline 1 & LR $3043 / 50$ & 5 & 5 & 0 & 7 & 60 \\
\hline 2 & LR $3043 / 50+30$ phr STX501 & 5 & 5 & 3 & 13 & 52 \\
\hline 3 & ELASTOSIL FILM 2030 250/50 & & & & & 50 \\
\hline
\end{tabular}

Table 3. Details of the Different Frame Types

\begin{tabular}{|c|c|c|c|c|c|}
\hline \multicolumn{2}{|c|}{ Name of frames } & $\begin{array}{l}\text { Size of the } \\
\text { frame (width } \\
\times \quad \text { length, } \\
\text { mm) }\end{array}$ & $\begin{array}{l}\text { Hole } \\
\text { size, } \\
\text { mm }\end{array}$ & $\begin{array}{l}\text { Number of } \\
\text { holes in the } \\
\text { frame }\end{array}$ & Example \\
\hline \multicolumn{2}{|l|}{ Type A } & $30 \times 92$ & 8 & 12 & :8 :8: \\
\hline \multirow{2}{*}{ Type B } & B1 & $30 \times 30$ & 10 & 1 & ) \\
\hline & B2 & $50 \times 50$ & 10 & 1 & $\begin{array}{l}\text { Same as B1, just a } \\
\text { bigger frame }\end{array}$ \\
\hline \multicolumn{2}{|l|}{ Type C } & $30 \times 30$ & 15 & 1 & $\begin{array}{l}\text { Same as B1, just a } \\
\text { bigger hole }\end{array}$ \\
\hline
\end{tabular}

dust particles or air bubbles). Imperfections are also shown to be minimized by prestretching. Furthermore, we demonstrate that the actuation mechanism is always oriented against the electrical field direction, while microscopic deformation is not driven by gravity. This leads us to a theoretical description for the actuation mechanism, explaining that electrostatic forces (negative corona) surrounding the electrode cause this actuation. These phenomena may explain the-very often encountered-large discrepancy between electrical breakdown strengths determined from static configurations compared to the true, dynamic dielectric elastomer actuators. Furthermore, this understanding opens up for designing materials with active surfaces.

\section{MATERIALS AND METHODS}

4.1. Sample Preparation. In this work, three different silicone elastomer samples are used. Samples 1 and 2 are based on the same commercial silicone elastomer, namely, Elastosil LR 3043/50. LR 3043/50 is a two-component liquid silicone rubber with short curing time and a relatively high viscosity (1200 mPa.s @ $1 \mathrm{~s}^{-1}$ ). In addition to that, ELASTOSIL LR $3043 / 50$ is relatively soft, have high tensile strain at break (462\%) and high breakdown strength $(145 \mathrm{~V} / \mu \mathrm{m})$. Sample 2 is reinforced with a $\mathrm{TiO}_{2} / \mathrm{SiO}_{2}$ filler, to improve the dielectric permittivity of the elastomer. The addition of inorganic filler causes reinforcing of the elastomer (Young's modulus, 8.27 $\mathrm{MPa}$ ), but the elastomer remains highly stretchable (tensile strain-457\%). Sample 3 is a commercially available silicone elastomer film, constructed from medical-grade silicone, and it is prepared in a clean room and in an optimized industrial process by Wacker Chemie AG. Sample 3 serves as a reference film with high purity and quality. A comparison of the dielectric and mechanical properties of the three samples can be found in Supporting Information Table S1.

- Sample 1 (Elastosil sample): ELASTOSIL LR 3043/50 A/B (Wacker Chemie AG, Germany) is a twocomponent liquid silicone rubber with a short curing time. Belsil solvent (Belsil DM 1 Plus, Wacker Chemie AG, Germany) is additionally used for sample preparation due to the high viscosity of the elastomer premixes.

- Sample 2 (sample with 30 parts per hundred rubber (phr) of filler): ELASTOSIL LR 3043/50 A/B (Wacker Chemie AG, Germany) and AEROXIDE STX $\mathrm{TiO}_{2} /$ $\mathrm{SiO}_{2}$ filler STX501 (Evonik Industries, Germany). This filler is a hydrophobized fumed silicon-titanium mixed oxide with a titanium dioxide core and a silica shell.

- Sample 3 (Elastosil sample prepared in company): ELASTOSIL FILM 2030 250/50 (Wacker Chemie AG, Germany) is a commercially available cross-linked silicone elastomer film.

Table 2 includes information on sample preparation and thickness. Sample 1 is prepared by mixing ELASTOSIL LR $3043 / 50$ part A and Belsil solvent for $2 \mathrm{~min}$ at $3000 \mathrm{rpm}$ in a SpeedMixer DAC 150 FVZ-K. Next, part B is added and the premix is mixed additionally for $2 \mathrm{~min}$ at $3000 \mathrm{rpm}$. To make sample 2, the STX501 filler is added into the premix of ELASTOSIL LR 3043/50 part A along with Belsil solvent in weights according to Table 2 , and they are mixed for $2 \mathrm{~min}$ at $3000 \mathrm{rpm}$. Thereafter, remaining filler aggregates are broken down with a spatula, and the mixture is then mixed for $1 \mathrm{~min}$ with a spatula and then subsequently mixed in the SpeedMixer for $2 \mathrm{~min}$ at $3000 \mathrm{rpm}$. Next, ELASTOSIL part B is added and mixed for $2 \mathrm{~min}$ at $3000 \mathrm{rpm}$. After mixing, uniform mixtures are coated with a film applicator (4340 Automatic Film 
Table 4. Details of the Different Types of Electrodes Used Within This Study

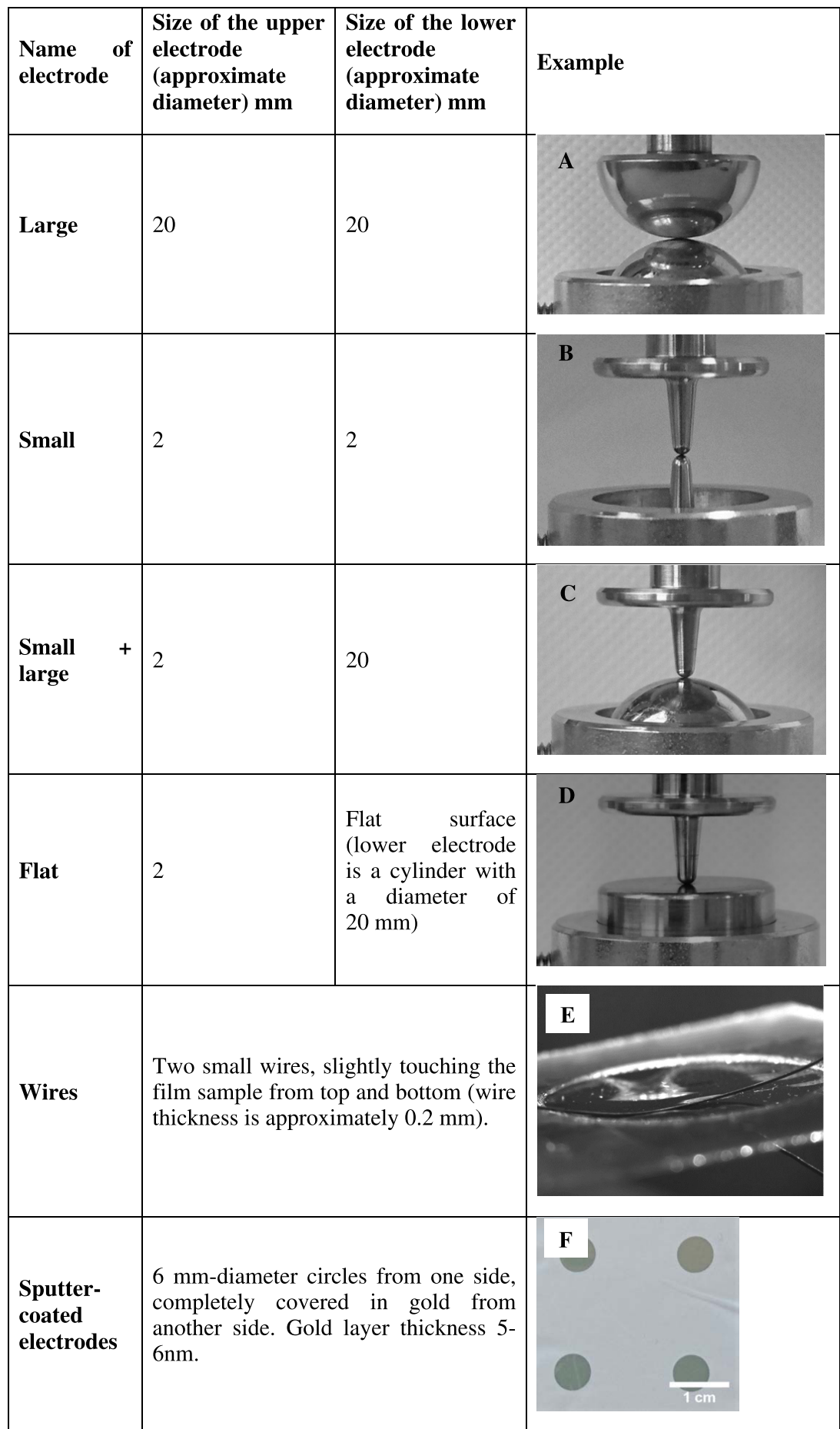

Applicator, Elcometer, Germany) with a $150 \mu \mathrm{m}$ gap on a glass substrate. First, coated samples are cured at $115{ }^{\circ} \mathrm{C}$ for an hour, following which postcuring is enabled by heating the cured film additionally at $200{ }^{\circ} \mathrm{C}$ for $4 \mathrm{~h} .{ }^{47,48}$ Sample 3 is supplied by Wacker Chemie AG as a $50 \mu \mathrm{m} \times 250 \mathrm{~mm} \times 300$ $\mathrm{mm}$ film sheet.

Film thickness is measured by cutting small $2 \mathrm{~mm} \times 7 \mathrm{~mm}$ strips out from several places on the film sheet. The crosssectional thickness of the strips is measured using a Leica DMLB optical microscope. At least three strips are tested, and the average film thickness is then calculated. The thicknesses of samples 1,2 , and 3 in this study are 60,52 , and $50 \mu \mathrm{m}$, respectively.

The expansion of the uniaxially stretched sample is described by the stretch ratio $\lambda$, and the material is assumed incompressible, so the sample thickness is calculated as $t=$ $t_{0} / \lambda$, where $t_{0}$ is the sample's initial thickness. ${ }^{34}$ The biaxially stretched sample expands radially by $\lambda$, and the thickness of the biaxially prestretched sample ${ }^{49}$ is calculated as $t=t_{0} / \lambda^{2}$.

4.2. Frames. To support the thin and soft elastomer films during the different measurements, a selection of frames is used, a list of which is presented in Table 3. Type A frame is 
made of a $0.5 \mathrm{~mm}$ thick polycarbonate sheet (RIAS A/S, Denmark), while types $\mathrm{B}$ and $\mathrm{C}$ are cut from a HOSTAPHAN polyester film (Pütz GmbH + Co. Folien KG, Germany) using a Yueming laser cutter (GD Han Syueming Laser Tech Co., Ltd., China).

The type A frame is used for breakdown measurements of the non-prestretched samples, and it is possible to take 12 measurements with one sample. Using this frame, measurements are performed with different kinds of electrodes (Table $4 \mathrm{~A}-\mathrm{D}$ ) in an in-house-built breakdown instrument (Pehama Productions A/S, Denmark, following the international standards IEC 60243-1 (1998) and IEC 60243-2 (2001) ${ }^{50}$ ). Type B frames are used for prestretched samples. Type B1 is for prestretching 10 or $20 \%$, and type B2 is for a $100 \%$ prestretch. The type $\mathrm{C}$ frame is used for breakdown measurements with wires and has a larger hole, to have a better view of the film during the actuation process.

4.3. Electrode Types. The different types of electrodes used in this work are presented in Table 4. Large semispherical steel electrodes (presented in Table 4A) are supplied by Pehama Productions A/S, Denmark, and small semispherical electrodes (Table 4B) are custom-made in our mechanical workshop. Sputter-coated gold electrodes (Table 4F) are prepared by completely coating one side of a film with a 5-6 $\mathrm{nm}$ thick gold layer and coating the other side with $6 \mathrm{~mm}$ diameter circles using a sputter coater (Q150T ES a turbomolecular-pumped high-resolution sputter coater, Quorum Technologies Ltd., United Kingdom; vacuum for $10 \mathrm{~s}$ and a $40 \mu \mathrm{A}$ current).

The electrodes A, B, C, and D (see Table 4 for further details) are used in the traditional breakdown setup, and electrode wires (Table 4E) are used in different setups by connecting them to a high-voltage power supply (model PS375, Stanford Research Systems, Inc.).

4.4. Biaxial Prestretch. The samples are cut into squares, and a circle is painted in the middle of the sample. The diameter of the circle depends on the prestretch ratio, so when the sample needs to be prestretched to 10,20 , or $100 \%$, the drawn circle diameters were $9.1,8.3$, and $5 \mathrm{~mm}$, respectively. The sample with the circle is placed onto the plastic type B frame and stretched to 10,20 , or $100 \%$ such that the drawn circle on the film becomes equal to the size of the frame hole $(10 \mathrm{~mm})$. The prestretched film is then fixed to the plastic frame with insulating tape (3M Scotch Crystal Tape, United Kingdom) on all sides. The biaxial prestretch process is presented in further detail in Supporting Information Figure S2.

4.4.1. Uniaxial Prestretch. The samples are cut into squares and placed onto the plastic frame. One side of the film is fixed to the frame with tape ( $3 \mathrm{M}$ Scotch Crystal Tape, United Kingdom), and the sample is then prestretched in the opposite direction of the tape by 10,20 , or $100 \%$, respectively. Subsequently, the stretched side of the film is fixed to the plastic frame with tape. A detailed scheme for how the sample is prestretched uniaxially is presented in more detail in Supporting Information Figure S3.

\section{EXPERIMENTAL SECTION}

A film sample is cut and placed onto the plastic frame, and together they are placed between two half-spherical-shaped electrodes (Figure 9a) or between two wires (Table 4E). The half-spherical (Table 4A-D) top electrode is lowered down to touch the elastomer, and the gap between electrodes is fixed at a certain preset with a micrometer stage and a gauge, depending on the thickness of the sample. When a wire electrode is used, the film sample is placed onto plastic frame type C (Table 3 ), which is fixed horizontally in the metallic holder, and two wires are attached from top and bottom (Table 4E). Double-sided adhesive tape (Fermoflex, Germany) is used with a flat electrode (Table 4D) to constrain the film sample.

To begin the experiment, voltage is ramped with $100 \mathrm{~V} / \mathrm{s}$ until the polymer film breaks down. The experiment is recorded using a high-speed camera system (FASTCAM Mini UX100, Photron) operating at $125 \mathrm{fps}$ and with a $1024 \times 1024$ pixel resolution.

The breakdown tests are carried out with both nonprestretched samples as well as with uniaxially and biaxially prestretched samples and gold sputter-coated samples. At least three samples are tested per configuration.

\section{ASSOCIATED CONTENT}

\section{Supporting Information}

The Supporting Information is available free of charge at https://pubs.acs.org/doi/10.1021/acsomega.0c00785.

Dielectric and mechanical sample properties (Table S1); bubble structure size and distance to the electrode (Table S2); calculated actuation strain (Figure S1); scheme for the biaxial sample's prestretching (Figure S2); scheme for the uniaxial sample's prestretching (Figure S3) (PDF)

Recording of a film stretching in a strongest electric field (MP4)

Recording of a bubble structure formation (MP4)

Recording of a ring-shaped structure formation (MP4) Coated gold burning (MP4)

\section{AUTHOR INFORMATION}

\section{Corresponding Author}

Anne Ladegaard Skov - Danish Polymer Centre, Technical

University of Denmark, Kgs. Lyngby 2800, Denmark;

○ orcid.org/0000-0003-1223-6638; Email: al@kt.dtu.dk

\section{Authors}

Justina Vaicekauskiate - Danish Polymer Centre, Technical University of Denmark, Kgs. Lyngby 2800, Denmark

Liyun Yu - Danish Polymer Centre, Technical University of Denmark, Kgs. Lyngby 2800, Denmark

Complete contact information is available at:

https://pubs.acs.org/10.1021/acsomega.0c00785

\section{Notes}

The authors declare no competing financial interest.

\section{ACKNOWLEDGMENTS}

The authors acknowledge funding from the Independent Research Fund Denmark.

\section{REFERENCES}

(1) Pelrine, R. E.; Kornbluh, R. D.; Joseph, J. P. Electrostriction of Polymer Dielectrics with Compliant Electrodes as a Means of Actuation. Sens. Actuators, A 1998, 64, 77-85.

(2) Palakodeti, R.; Kessler, M. R. Influence of Frequency and Prestrain on the Mechanical Efficiency of Dielectric Electroactive Polymer Actuators. Mater. Lett. 2006, 60, 3437-3440. 
(3) Koh, S. J. A.; Li, T.; Zhou, J.; Zhao, X.; Hong, W.; Zhu, J.; Suo, Z. Mechanisms of Large Actuation Strain in Dielectric Elastomers. J. Polym. Sci., Part B: Polym. Phys. 2011, 49, 504-515.

(4) Zhao, X.; Suo, Z. Theory of Dielectric Elastomers Capable of Giant Deformation of Actuation. Phys. Rev. Lett. 2010, 104, No. 178302.

(5) Carpi, F.; Bauer, S.; Rossi, D. De. Stretching Dielectric Elastomer Performance. Science 2010, 330, 1759-1761.

(6) Baumgartner, R.; Keplinger, C.; Kaltseis, R.; Schwödiauer, R.; Bauer, S. In Dielectric Elastomers: From the Beginning of Modern Science to Applications in Actuators and Energy Harvesters, Proceedings of SPIE, Electroactive Polymer Actuators and Devices (EAPAD) 2011; 797603.

(7) Keplinger, C.; Kaltenbrunner, M.; Arnold, N.; Bauer, S. Röntgen's Electrode-Free Elastomer Actuators without Electromechanical Pull-in Instability. Proc. Natl. Acad. Sci. U.S.A. 2010, 107, 4505-4510.

(8) Li, P.; Wang, Y.; Gupta, U.; Liu, J.; Zhang, L.; Du, D.; Foo, C. C.; Ouyang, J.; Zhu, J. Transparent Soft Robots for Effective Camouflage. Adv. Funct. Mater. 2019, 29, No. 1901908.

(9) Chen, Y.; Zhao, H.; Mao, J.; Chirarattananon, P.; Helbling, E. F.; Hyun, N. P.; Clarke, D. R.; Wood, R. J. Controlled Flight of a Microrobot Powered by Soft Artificial Muscles. Nature 2019, 575, 324-329.

(10) Sirbu, I.; Moretti, G.; Dire, S.; Fambri, L.; Vertechy, R.; Meniglio, D.; Fontana, M. Electrostatic Actuator for Tactile Display Based on Hydraulically Coupled Dielectric Fluids and Soft Structures. Proc. SPIE 2019, 10966, 1-6.

(11) Yun, S.; Park, S.; Park, B.; Ryu, S.; Jeong, S. M.; Kyung, K.-U. A Soft and Transparent Visuo-Haptic Interface Pursuing Wearable Devices. IEEE Trans. Ind. Electron. 2020, 67, 717-724.

(12) Zhang, H.; Dai, M.; Zhang, Z. The Analysis of Transparent Dielectric Elastomer Actuators for Lens. Optik 2019, 178, 841-845.

(13) Qiu, Y.; Zhang, E.; Plamthottam, R.; Pei, Q. Dielectric Elastomer Artificial Muscle: Materials Innovations and Device Explorations. Acc. Chem. Res. 2019, 52, 316-325.

(14) Blok, J.; LeGrand, D. G. Dielectric Breakdown of Polymer Films. J. Appl. Phys. 1969, 40, 288-293.

(15) Madsen, F. B.; Yu, L.; Mazurek, P.; Skov, A. L. A Simple Method for Reducing Inevitable Dielectric Loss in High-Permittivity Dielectric Elastomers. Smart Mater. Struct. 2016, 25, No. 075018.

(16) Zakrevskiil, V. A. Electrical Breakdown of Thin Polymer Films. Phys. Solid State 2005, 47, 961-967.

(17) Carpi, F.; Anderson, I.; Bauer, S.; Frediani, G.; Gallone, G.; Gei, M.; Graaf, C.; Jean-Mistral, C.; Kaal, W.; Kofod, G.; et al. Standards for Dielectric Elastomer Transducers. Smart Mater. Struct. 2015, 24, No. 105025.

(18) Seelecke, S.; Welsch, F.; Fasolt, B. In Dielectric Breakdown Test Setup for Dielectric Elastomers: Design and Validation, Proceedings of SPIE, 2018.

(19) Belkin, A.; Bezryadin, A.; Hendren, L.; Hubler, A. Recovery of Alumina Nanocapacitors after High Voltage Breakdown. Sci. Rep. 2017, 7, No. 932.

(20) Greaney, P.; Meere, M.; Zurlo, G. The Out-of-Plane Behaviour of Dielectric Membranes: Description of Wrinkling and Pull-in Instabilities. J. Mech. Phys. Solids 2019, 122, 84-97.

(21) Plante, J. S.; Dubowsky, S. Large-Scale Failure Modes of Dielectric Elastomer Actuators. Int. J. Solids Struct. 2006, 43, 77277751.

(22) Zhao, X.; Suo, Z. Method to Analyze Electromechanical Stability of Dielectric Elastomers. Appl. Phys. Lett. 2007, 91, No. 061921.

(23) Li, X.; Tung, C. H.; Pey, K. L. The Nature of Dielectric Breakdown. Appl. Phys. Lett. 2008, 93, No. 072903.

(24) Zurlo, G.; Destrade, M.; DeTommasi, D.; Puglisi, G. Catastrophic Thinning of Dielectric Elastomers. Phys. Rev. Lett. 2017, 118, No. 078001.
(25) Zhao, X.; Wang, Q. Harnessing Large Deformation and Instabilities of Soft Dielectrics: Theory, Experiment, and Application. Appl. Phys. Rev. 2014, 1, No. 021304.

(26) Pelrine, R.; Kornbluh, R. Dielectric Elastomers as Electroactive Polymers (EAPs): Fundamentals, 1st ed.; Carpi, F., Ed.; Springer International Publishing: Switzerland, 2016.

(27) Mateiu, R. V.; Yu, L.; Skov, A. L. In Electrical Breakdown Phenomena of Dielectric Elastomers, Proceedings of SPIE, 2017.

(28) Förster-Zügel, F.; Solano-Arana, S.; Klug, F.; Schlaak, H. F. Dielectric Breakdown Strength Measurements with Silicone-Based Single-Layer Dielectric Elastomer Transducers. Smart Mater. Struct. 2019, 28, No. 075042.

(29) Godaba, H.; Zhang, Z. Q.; Gupta, U.; Foo, C. C.; Zhu, J. Instabilities in Dielectric Elastomers: Buckling, Wrinkling, and Crumpling. Soft Matter 2019, 15, 7137-7144.

(30) Godaba, H.; Zhang, Z. Q.; Gupta, U.; Chiang Foo, C.; Zhu, J. Dynamic Pattern of Wrinkles in a Dielectric Elastomer. Soft Matter 2017, 13, 2942-2951.

(31) Li, T.; Keplinger, C.; Baumgartner, R.; Bauer, S.; Yang, W.; Suo, Z. Giant Voltage-Induced Deformation in Dielectric Elastomers near the Verge of Snap-through Instability. J. Mech. Phys. Solids 2013, 61, 611-628.

(32) Christensen, L. R.; Hassager, O.; Skov, A. L. Electro-Thermal Model of Thermal Breakdown in Multilayered Dielectric Elastomers. AIChE J. 2019, 65, 859-864.

(33) Kleo, M.; Förster-Zügel, F.; Schlaak, H. F.; Wallmersperger, T. Thermo-Electro-Mechanical Behavior of Dielectric Elastomer Actuators: Experimental Investigations, Modeling and Simulation. Smart Mater. Struct. 2020, 29, No. 085001.

(34) Zakaria, S.; Yu, L.; Kofod, G.; Skov, A. L. The Influence of Static Pre-Stretching on the Mechanical Ageing of Filled Silicone Rubbers for Dielectric Elastomer Applications. Mater. Today Commun. 2015, 4, 204-213.

(35) Bigué, J. L.; Chouinard, P.; Proulx, S.; Miron, G.; Plante, J. S. Preliminary Assesment of Manufacturing Impacts on Dielectric Elastomer Actuators Reliability, CanSmart2009; International Workshop on Smart Materials and Structures, 2009; pp 303-314.

(36) Silau, H.; Stabell, N. B.; Petersen, F. R.; Pham, M.; Yu, L.; Skov, A. L. Weibull Analysis of Electrical Breakdown Strength as an Effective Means of Evaluating Elastomer Thin Film Quality. Adv. Eng. Mater. 2018, 20, No. 1800241.

(37) Yu, L.; Skov, A. L. Molecular Strategies for Improved Dielectric Elastomer Electrical Breakdown Strengths. Macromol. Rapid Commun. 2018, 39, No. 1800383.

(38) Fasolt, B.; Welsch, F.; Jank, M.; Seelecke, S. Effect of Actuation Parameters and Environment on the Breakdown Voltage of Silicone Dielectric Elastomer Films. Smart Mater. Struct. 2019, 28, No. 094002.

(39) Dissado, L. A.; Fothergill, J. C. Electrical Degradation and Breakdown in Polymers, 1st ed.; Stevens, G. C., Ed.; The Institution of Engineering and Technology: London, United Kingdom, 1992.

(40) Raizer, Y. P. Gas Discharge Physics, 2nd ed.; Allen, J. E., Ed.; Springer-Verlag Berlin Heidelberg: Moscow, Russia, 1992.

(41) Dhawan, A.; Muth, J. F. Integration of Nanoparticles into and onto Optical Fiber Sensors Mater. Res. Soc. Symp. Proc. 2005, 900, DOI: 10.1557/PROC-0900-O11-09.

(42) Reiser, A.; Lock, M. W. B.; Knight, J. Migration and Trapping of Extrinsic Charge Carriers in Polymer Films. Trans. Faraday Soc. 1969, 65, 2168-2185.

(43) Ashter, S. A. Thermoforming of Single and Multilayer Laminates. Plastic Films Technologies, Testing, and Applications, 2nd ed.; Ebnesajjad, S., Ed.; William Andrew Publishing: Norwich, United States, 2014.

(44) Li, B.; Chen, H.; Qiang, J.; Hu, S.; Zhu, Z.; Wang, Y. Effect of Mechanical Pre-Stretch on the Stabilization of Dielectric Elastomer Actuation. J. Phys. D. Appl. Phys. 2011, 44, No. 155301.

(45) Iannarelli, A.; Niasar, M. G.; Ross, R. The Effects of Static PreStretching on the Short and Long-Term Reliability of Dielectric Elastomer Actuators. Smart Mater. Struct. 2019, 28, No. 125014. 
(46) Zhou, J.; Hong, W.; Zhao, X.; Zhang, Z.; Suo, Z. Propagation of Instability in Dielectric Elastomers. Int. J. Solids Struct. 2008, 45, $3739-3750$.

(47) Zakaria, S.; Madsen, F. B.; Skov, A. L. Post Curing as an Effective Means of Ensuring the Long-Term Reliability of PDMS Thin Films for Dielectric Elastomer Applications. Polym.-Plast. Technol. Eng. 2017, 56, 83-95.

(48) Brook, M. A.; Saier, H. U.; Schnabel, J.; Town, K.; Maloney, M. Pretreatment of Liquid Silicone Rubbers to Remove Volatile Siloxanes. Ind. Eng. Chem. Res. 2007, 46, 8796-8805.

(49) Zakaria, S.; Morshuis, P. H. F.; Benslimane, M. Y.; Yu, L.; Skov, A. L. The Electrical Breakdown Strength of Pre-Stretched Elastomers, with and without Sample Volume Conservation. Smart Mater. Struct. 2015, 24, No. 055009.

(50) Vudayagiri, S.; Zakaria, S.; Yu, L.; Hassouneh, S. S.; Benslimane, M.; Skov, A. L. High Breakdown-Strength Composites from Liquid Silicone Rubbers. Smart Mater. Struct. 2014, 23, No. 105017. 